\title{
ON DIRICHLET SERIES ASSOCIATED WITH POLYNOMIALS
}

\author{
E. CARLETTI AND G. MONTI BRAGADIN
}

(Communicated by William Adams)

\begin{abstract}
Let $P(X)$ be a polynomial of degree $N$ with complex coefficients and $d_{1}, d_{2}$ two complex numbers with real part greater then -1 . Consider the Dirichlet series associated with the triple $\left(P(X), d_{1}, d_{2}\right)$

$$
L(s)=\sum_{n=1}^{\infty} \frac{P(n)}{\left(n+d_{1}\right)^{s}\left(n+d_{2}\right)^{s}} .
$$

In this paper we get an explicit formula for $L(s)$ in terms of special functions which gives meromorphic continuation of $L(s)$ with at most simple poles at $s=(N+1-k) / 2, k=0,1, \ldots$. Finally we apply our explicit formula to Minakshisundaram's zeta function of the three-dimensional sphere.
\end{abstract}

\section{INTRODUCTION}

Mellin first studied the analytic continuation of Dirichlet series associated with one polynomial of several variables in [6] (see also [3]). We are concerned with Dirichlet series of the form $\sum_{n=0}^{\infty} P(n) Q(n)^{-s}$, where $P(n)$ and $Q(n)$ are two polynomials of one variable. The aim of this paper is to obtain meromorphic continuation of Dirichlet series of the form

$$
L(s)=\sum_{n=1}^{\infty} \frac{P(n)}{\left(n+d_{1}\right)^{s}\left(n+d_{2}\right)^{s}}
$$

where $d_{1}, d_{2}$ are two complex numbers with $\Re\left(d_{i}\right)>-1, i=1,2$, and $P(n)$ is a polynomial of degree $N$. The above series converges absolutely in the half plane $\Re(s)>(N+1) / 2$, where it represents an analytic function. In [8] Eie obtains an analytic continuation for Dirichlet series of the type:

$$
\sum_{n=1}^{\infty} \frac{1}{\left[\left(n+d_{1}\right)\left(n+d_{2}\right) \cdots\left(n+d_{k}\right)\right]^{s}}
$$

by giving an integral expression (see $[8$, p. 586]). In our paper after obtaining a similar integral expression we succeed in getting an explicit formula by means of special functions. This gives the analytic continuation.

Received by the editors November 14, 1991 and, in revised form, August 14, 1992.

1991 Mathematics Subject Classification. Primary 11 M41. 
Remarkable examples of our Dirichlet series are Minakshisundaram's zeta functions $\sum_{n=1}^{\infty} \lambda_{n}^{-s}$, where the $\lambda_{n}$ 's are the eigenvalues of the Laplace operator on the canonical spaceforms (real spheres, real and complex projective spaces), (see [2, pp. 146-178]) so that we obtain a proof of their analytic continuation involving only tools of complex analysis (for different methods see [7, 4]).

We get the following result.

Theorem. Let $L(s)$ be defined by (1), where $d_{1}, d_{2}$ are two complex numbers with $\Re\left(d_{i}\right)>-1, i=1,2$, and $P(n)$ is a polynomial of degree $N$. Then $L(s)$ has a meromorphic continuation on the complex plane with at most simple poles at $s=(N+1-k) / 2, k=0,1,2, \ldots$.

We deduce our result from formula (5) which is in turn obtained by a direct computation. The key point in our proof is the lemma below that generalizes a well-known formula (see [5, no. 3411-7]). Finally in $\S 3$ we apply (5) to Minakshisundaram's zeta function of the sphere $S^{3}$.

\section{PROOF OF THE THEOREM}

Lemma. Let $m$ be a nonnegative integer, $\alpha$ and $\beta$ be complex numbers such that $\Re(\alpha)>m, \Re(\beta)<m+1$, and

$$
I(\alpha, \beta, m)=\int_{0}^{\infty} \frac{e^{\beta t} t^{\alpha}}{\left(e^{t}-1\right)^{m+1}} d t .
$$

Then

$$
I(\alpha, \beta, m)=\Gamma(\alpha+1) \sum_{j=0}^{m} P_{m-j}^{m}(\beta) \zeta(\alpha+1-j, m-\beta+1)
$$

where $P_{m-j}^{m}(\beta)$ are polynomials in $\beta$ of degree $m-j$ defined by

$$
\begin{gathered}
P_{0}^{0}(\beta)=1, \quad P_{m}^{m}(\beta)=\frac{1}{m !} \prod_{k=1}^{m}(\beta-k), \\
P_{m-j}^{m}(\beta)=\frac{1}{j !} \frac{d^{j}}{d \beta^{j}} P_{m}^{m}(\beta), \quad 0 \leq j \leq m,
\end{gathered}
$$

and $\zeta(s, a)$ is the Hurwitz zeta function.

Proof. $I(\alpha, \beta, m)$ is clearly absolute convergent, and integrating by parts we have for $m \geq 1$ that

$$
I(\alpha, \beta, m)=\frac{1}{m}[\alpha I(\alpha-1, \beta-1, m-1)+(\beta-1) I(\alpha, \beta-1, m-1)] .
$$

Since

$$
I(\alpha, \beta, 0)=\Gamma(\alpha+1) \zeta(\alpha+1,1-\beta)
$$

(see [5, no. 3411-7]), the result follows by induction.

We observe that

$$
m ! P_{m}^{m}(\beta)=\sum_{k=1}^{m+1} s_{m+1, k} \beta^{k-1}
$$

where $s_{m+1, k}$ are the Stirling numbers of the first kind (see [1, Chapter III, pp. 88-93]). 
Now we prove the theorem. Let us consider the series

$$
L_{m}(s)=\sum_{n=1}^{\infty} \frac{n^{m}}{\left(n+d_{1}\right)^{s}\left(n+d_{2}\right)^{s}}
$$

It converges absolutely (and uniformly on compact subsets) in $\Re(s)>(1+m) / 2$. If $P(n)=\sum_{m=0}^{N} a_{m} n^{m}$, we have

$$
L(s)=\sum_{m=0}^{N} a_{m} L_{m}(s)
$$

Starting from well-known formulas

$$
\int_{0}^{\infty} y_{i}^{s-1} e^{-\left(n+d_{i}\right) y_{i}} d y_{i}=\Gamma(s)\left(n+d_{i}\right)^{-s}, \quad i=1,2
$$

by multiplying these integrals, and summing on $n$ we get

$$
\begin{aligned}
L_{m}(s) \Gamma(s)^{2} & =\int_{0}^{\infty} \int_{0}^{\infty} \sum_{n=1}^{\infty} n^{m} e^{-n\left(y_{1}+y_{2}\right)}\left(y_{1} y_{2}\right)^{s-1} e^{-d_{1} y_{1}-d_{2} y_{2}} d y_{1} d y_{2} \\
& =\int_{0}^{\infty} \int_{0}^{\infty} \frac{P_{m}\left(e^{-\left(y_{1}+y_{2}\right)}\right)}{\left(1-e^{-\left(y_{1}+y_{2}\right)}\right)^{m+1}}\left(y_{1} y_{2}\right)^{s-1} e^{-d_{1} y_{1}-d_{2} y_{2}} d y_{1} d y_{2}
\end{aligned}
$$

In fact (see [1, Chapter V]), we have $\sum_{n=1}^{\infty} n^{m} z^{n}=P_{m}(z) /(1-z)^{m+1}$ where $P_{0}(z)=z$ and, if $m \neq 0, P_{m}(z)=\sum_{p=1}^{m} a_{p}^{m} z^{p}$ with

$$
a_{p}^{m}=\sum_{l=1}^{p}(-1)^{p-l}\left(\begin{array}{c}
m+1 \\
p-l
\end{array}\right) l^{m}
$$

Now we use the substitution

$$
\begin{cases}y_{1}=t_{1} t_{2}, & t_{1} \in(0,+\infty) \\ y_{2}=t_{1}\left(1-t_{2}\right), & t_{2} \in(0,1)\end{cases}
$$

so that, setting $\delta=d_{2}-d_{1}$ and $\Delta=\left(d_{2}+d_{1}\right) / 2$, by [5, no. 3383-2], we get

$$
\begin{aligned}
L_{m}(s) \Gamma(s)^{2}= & \int_{0}^{\infty} \int_{0}^{1} \frac{P_{m}\left(e^{-t_{1}}\right)}{\left(1-e^{-t_{1}}\right)^{m+1}} t_{1}^{2 s-1} t_{2}^{s-1}\left(1-t_{2}\right)^{s-1} e^{\delta t_{1} t_{2}-d_{2} t_{1}} d t_{1} d t_{2} \\
= & \int_{0}^{\infty} \frac{P_{m}\left(e^{-t_{1}}\right)}{\left(1-e^{-t_{1}}\right)^{m+1}} e^{-d_{2} t_{1}} t_{1}^{2 s-1} \int_{0}^{1} e^{\delta t_{1} t_{2}} t_{2}^{s-1}\left(1-t_{2}\right)^{s-1} d t_{2} d t_{1} \\
= & \sqrt{\pi} \int_{0}^{\infty} \frac{P_{m}\left(e^{-t_{1}}\right)}{\left(1-e^{-t_{1}}\right)^{m+1}} t_{1}^{2 s-1} t_{1}^{1 / 2-s} \delta^{1 / 2-s} e^{-d_{2} t_{1}+t_{1} \delta / 2} \\
& \times \Gamma(s) I_{s-1 / 2}\left(\frac{t_{1} \delta}{2}\right) d t_{1},
\end{aligned}
$$

where $I_{s-1 / 2}(z)$ is the Bessel function of the first kind. Hence by [5, no. 8445] 
and the lemma, for $m \geq 1$ we have that

(3)

$$
\begin{aligned}
& L_{m}(s) \Gamma(s)=\sqrt{\pi} \delta^{1 / 2-s} \int_{0}^{\infty} \frac{P_{m}\left(e^{-t}\right)}{\left(1-e^{-t}\right)^{m+1}} t^{s-1 / 2} e^{-d_{2} t+\delta t / 2} I_{s-1 / 2}\left(\frac{t \delta}{2}\right) d t \\
& =\sqrt{\pi} 4^{1 / 2-s} \int_{0}^{\infty} \frac{P_{m}\left(e^{-t}\right)}{\left(1-e^{-t}\right)^{m+1}} t^{2 s-1} e^{-d_{2} t+\delta t / 2} \sum_{l=0}^{\infty}\left(\frac{\delta}{4}\right)^{2 l} \frac{t^{2 l}}{l ! \Gamma(s+l+1 / 2)} d t \\
& =\sqrt{\pi} 4^{1 / 2-s} \sum_{l=0}^{\infty}\left(\frac{\delta}{4}\right)^{2 l} \frac{1}{l ! \Gamma(s+l+1 / 2)} \int_{0}^{\infty} \frac{P_{m}\left(e^{-t}\right)}{\left(e^{t}-1\right)^{m+1}} t^{2 s+2 l-1} e^{t(m+1-\Delta)} d t \\
& =\sqrt{\pi} 4^{1 / 2-s} \sum_{l=0}^{\infty}\left(\frac{\delta}{4}\right)^{2 l} \frac{1}{l ! \Gamma(s+l+1 / 2)} \sum_{p=1}^{m} a_{p}^{m} \int_{0}^{\infty} \frac{e^{t(m+1-\Delta-p)}}{\left(e^{t}-1\right)^{m+1}} t^{2 s+2 l-1} d t \\
& =\sqrt{\pi} 4^{1 / 2-s} \sum_{j=0}^{m} \sum_{p=1}^{m} \sum_{l=0}^{\infty}\left(\frac{\delta}{4}\right)^{2 l} \frac{\Gamma(2 s+2 l) a_{p}^{m}}{l ! \Gamma(s+l+1 / 2)} P_{m-j}^{m}(m+1-p-\Delta) \\
& =\sum_{j=0}^{m} \sum_{p=1}^{m} \sum_{l=0}^{\infty}\left(\frac{\delta}{2}\right)^{2 l} \frac{\Gamma(s+l) a_{p}^{m}}{l !} P_{m-j}^{m}(m+1-p-\Delta) \\
& \times \zeta(2 s+2 l-j, p+\Delta) .
\end{aligned}
$$

If $m=0$, we have

$$
\begin{aligned}
L_{0}(s) \Gamma(s) & =\sqrt{\pi} 4^{1 / 2-s} \sum_{l=0}^{\infty}\left(\frac{\delta}{4}\right)^{2 l} \frac{1}{l ! \Gamma(s+l+1 / 2)} \int_{0}^{\infty} \frac{e^{-\Delta t}}{\left(e^{t}-1\right)} t^{2 s+2 l-1} d t \\
& =\sqrt{\pi} 4^{1 / 2-s} \sum_{l=0}^{\infty}\left(\frac{\delta}{4}\right)^{2 l} \frac{\Gamma(2 s+2 l)}{l ! \Gamma(s+l+1 / 2)} \zeta(2 s+2 l, 1+\Delta) .
\end{aligned}
$$

Hence from (2)-(4) and well-known formulas for the $\Gamma$-function we get

$$
\begin{aligned}
L(s)= & \sum_{l=0}^{\infty}\left(\frac{\delta}{2}\right)^{2 l}(-1)^{l}\left(\begin{array}{c}
-s \\
l
\end{array}\right) \\
\times & {\left[a_{0} \zeta(2 s+2 l, 1+\Delta)\right.} \\
& \left.\quad+\sum_{m=1}^{N} \sum_{j=0}^{m} \sum_{p=1}^{m} a_{m} a_{p}^{m} P_{m-j}^{m}(m+1-p-\Delta) \zeta(2 s+2 l-j, p+\Delta)\right] .
\end{aligned}
$$

The above formula and the meromorphic continuation of Hurwitz zeta function give the meromorphic continuation over $\mathbb{C}$ of $L(s)$ with simple poles at most at $s=(N+1-k) / 2$, where $k$ runs through nonnegative integers, since

$$
\sum_{j=0}^{m} \sum_{l=0}^{\infty}\left(\frac{\delta}{2}\right)^{2 l}(-1)^{l}\left(\begin{array}{c}
-s \\
l
\end{array}\right) \zeta(2 s+2 l-j, p+\Delta)
$$


is an absolutely (uniformly on compact subsets) convergent series on the set $\mathbb{C} \backslash\left\{(N+1-k) / 2, k \in \mathbb{Z}^{+}\right\}$.

\section{AN APPLICATION}

Let us denote Minakshisundaram's zeta function of $S^{3}$ by $Z_{3}(s)$ so that, according to [2],

$$
Z_{3}(s)=\sum_{n=1}^{\infty} \frac{n^{2}+2 n+1}{[n(n+2)]^{s}}
$$

The values of parameters for $Z_{3}(s)$ in $(5)$ are

$$
\begin{gathered}
N=2,1 \leq m \leq 2, a_{0}=1, a_{1}=2, a_{2}=1, a_{1}^{1}=a_{2}^{2}=a_{1}^{2}=1, \\
d_{1}=0, d_{2}=2, \frac{1}{2} \delta=1, \Delta=1, \\
P_{1}^{1}(\beta)=\beta-1, P_{0}^{1}(\beta)=1, P_{2}^{2}(\beta)=\frac{1}{2}(\beta-1)(\beta-2), \\
P_{1}^{2}(\beta)=\beta-\frac{3}{2}, P_{0}^{2}(\beta)=\frac{1}{2} .
\end{gathered}
$$

By reducing all Hurwitz zeta functions to $\zeta(-, 2)$ a straightforward computation gives

$$
Z_{3}(s)=\sum_{l=0}^{\infty}(-1)^{l}\left(\begin{array}{c}
-s \\
l
\end{array}\right) \zeta(2 s+2 l-2,2) .
$$

From above it follows that the poles of $Z_{3}(s)$ are exactly the points $s=\frac{3}{2}-$ $n, n=0,1, \ldots$, and they are all simple. Moreover,

$$
\operatorname{Res}\left(Z_{3}(s), \frac{3}{2}-n\right)=(-1)^{n} \frac{(2 n-3) ! !}{2^{n} n !}
$$

One should compare this way to obtain the residues of $Z_{3}(s)$ with that given in the general case (see [4, p. 79]). Finally we note that $Z_{3}(-n)=0, n=$ $0,1, \ldots$.

\section{REFERENCES}

1. M. Aigner, Combinatorial theory, Springer, New York, 1979.

2. M. Berger, P. Gauduchon, and E. Mazet, Le spectre d'une variété riemannienne, Springer, New York, 1971.

3. P. Cassou-Noguès, Dirichlet series associated with a polynomial, Lecture Notes in Phys., vol. 47, Springer, New York, 1990, pp. 244-252.

4. P. Gilkey, Invariance theory. The heat equation and the Atiyah-Singer index theorem, Publish or Perish, Cambridge, MA, 1984.

5. I. S. Gradshteyn and I. M. Ryzhik, Table of integrals, series and products, Academic Press, New York, 1980.

6. H. Mellin, Eine Formel für den Logarithmus transzendenter Funktionen von endlichem Geschlecht, Acta Soc. Sci. Fennicae 29 (1900), 3-49.

7. S. Minakshisundaram, Zeta functions on the sphere, J. Indian Math. Soc. 13 (1949), 41-48.

8. Minking Eie, On a Dirichlet series associated with a polynomial, Proc. Amer. Math. Soc. 110 (1990), 583-590.

Dipartimento di Matematica, University degli Studi, Via L. B. Alberti 4, 16132 GenOVA, ITALY 